Viszeralmedizin 25 | 4 | 09

Rash in der Therapie des metastasierten Pankreaskarzinoms: Management einer "positiven Nebenwirkung"

Tritt unter der Kombinationstherapie Gemcitabin und Erlotinib (Tarceva ${ }^{\circledR}$ ) bei Patienten mit fortgeschrittenem Pankreaskarzinom ein akneähnlicher Hautausschlag (Rash) auf, ist das ein positives Zeichen: Ergebnissen internationaler PhaseIII-Studien zufolge sprechen diese Patienten überproportional gut auf die Behandlung an [1, 2]. Die kutanen Nebenwirkungen verändern sich meist mit der Zeit. So beginnt die Erstmanifestation überwiegend in den seborrhoischen Arealen an Gesicht, Hals, Schulter und am oberen Stamm. Auch Rhagaden an den Finger- und Zehenkuppen und die - seltener auftretenden - Paronychien an Hand- und Fußnägeln zählen zu den frühen Hautveränderungen. $\mathrm{Ab}$ der zweiten $\mathrm{Be}$ handlungswoche zeigt sich oft eine trockene und schuppende Haut. Viele Patienten klagen über mehr oder weniger starken Juckreiz. In seltenen Fällen können nach einigen Monaten eine diffuse Alopezie oder Veränderungen von Wimpern und Augenbrauen auftreten. Allerdings beeinträchtigen diese Hautveränderungen Studienergebnissen zufolge kaum die Lebensqualität der Patienten. Nichtsdestotrotz reduzieren die behandelnden Ärzte häufig aufgrund fehlender Erfahrungen mit dermatologischen Erkrankungen die Erlotinib-Dosis oder unterbrechen die Therapie, sobald ein Rash auftritt. Um niedergelassenen Onkologen, Pneumologen und Gastroenterologen sowie Kliniken mit ambulanter Versorgung Sicherheit im Umgang mit den Erlotinib-induzierten Hautreaktionen zu bieten, hat die Roche Pharma AG eine umfangreiche Kampagne ins Leben gerufen. Sie umfasst beispielsweise Kurzschulungen zum Rash-Management auf der Basis eines Konzepts von Professor Bernhard
Homey aus der Hautklinik des Universitätsklinikums Düsseldorf. Innerhalb von 90 min erfahren die Behandlungsteams wichtige Aspekte der Diagnostik, Prävention und Therapie der verschiedenen kutanen Nebenwirkungen. Interessierte Ärzte können auf der Webseite der Roche Pharma AG einen Termin für eine Rash-Management-Schulung buchen. Informationen dazu erhalten sie über den Roche Außendienst.

\section{Referenzen}

1 Moore MJ et al.: J Clin Oncol 2007;25:1960-1966.

2 Verslype C et al.: ASCO 2009. J Clin Oncol 2009;27:15s (abstr 4532).

Weitere Informationen bei

Roche Pharma AG

Dr. H.-U. Jelitto

Emil-Barell-Straße 1, 79639 Grenzach-Wyhlen Tel.+49 7624 14-3715, Fax -3366

www.roche.de

\section{Spitzenplatz in der Bewertung durch das US Patent Board: Covidien weiter vorne}

Auch in seiner neuesten Veröffentlichung vom 14. Oktober 2009 berichtete das Patent Board in Chicago (USA), dass Covidien weiterhin die Branche in Sachen Innovationskraft anführt [1]. Basis der Analysen des Patent Board ist die Bewertung der jeweiligen Patent-Portfolios von insgesamt mehr als 100 internationalen Firmen. Besonders bemerkenswert fand das Board diesmal den Zugewinn Covidiens beim Indikator «Science Strength» (+48\%), der den engen Bezug des Unternehmens zu wissenschaftlichen Forschungen widerspiegelt. Und auch beim Indikator «Technology Strength» hat Covidien deutlich zugelegt $(+15 \%)$. Insbesondere in der Chirurgie zählt Covidien mit seinen renommierten Marken AutoSuture ${ }^{\mathrm{TM}}$, Valley Lab ${ }^{\mathrm{TM}}$, Sofra-
$\operatorname{dim}^{\mathrm{TM}}$ oder Syneture ${ }^{\mathrm{TM}}$ seit vielen Jahren zu den unangefochtenen Marktführern. Aktuellste Beispiele der Innovationskraft von Covidien in der Chirurgie sind das Klammernahtmagazin Duet TRS $^{\text {TM }}$ (Tissue Reinforcement System), die mit enorm positivem Feedback angenommenen Entwicklungen im Bereich SILS ${ }^{\mathrm{TM}}$ (Single Incision Laparoscopic Surgery) oder auch das höchst innovative Nahtmaterial der V-loc ${ }^{\mathrm{TM}}$ Produktserie. Das Ziel der globalen Aktivitäten von Covidien ist es, evidenzbasierte Lösungen und Dienstleistungen anzubieten, die dazu beitragen, medizinische Verfahren zum Nutzen der Patienten weiter zu verbessern, therapeutische Komplikationen zu verringern und die Behandlungskosten im Gesundheitswesen durch geeignete Präventions- und Sicherheitsmaßnahmen zu reduzieren. Dabei setzt Covidien auf eine weltweite und enge Zusammenarbeit mit medizinischen Fachleuten aller Gesundheitsbe- reiche. Covidien produziert und vertreibt seine High-End-Medizinprodukte in den Segmenten «Offene und Minimalinvasive Chirurgie», «Beatmungs- und Intensivmedizin», «Diagnostik» sowie «Krankenpflege» in mehr als 140 Ländern der Erde.

\section{Referenz}

1 The Patent Board, 20 North Wacker Drive, Chicago, IL 60606. http://www patentboard.com/PressRoom/Article View/tabid/95/smid/440/ArticleID/108/reftab/38/t/ Covidien $\% 20$ continues $\% 20$ to $\% 20$ lead $\% 20$ industry/ Default.aspx.

Weitere Informationen bei Covidien Deutschland GmbH Pressesprecher Dr. Jörg Kunz Tel.+49 1733692693

Joerg.kunz@covidien.com www.covidien.com

\title{
Ticker+++ Ticker+++ Ticker+++ Ticker+++ Ticker+++ Ticker+++ Ticker+++
}

Essex Pharma GmbH. In der Medikamentengruppe der Biologics nimmt REMICADE ${ }^{\circledR}$ (Infliximab) von Essex Pharma seit Jahren eine hohe Stellung ein. Das gilt zum einen hinsichtlich des guten Wirksamkeits- und Verträglichkeitsprofils, zum anderen aber auch wegen der äußerst weit gesteckten Einsatzmöglichkeiten dieses Vorreiters der Anti-TNF-Therapie. Aus diesem Grund wurde REMICADE ${ }^{\circledR}$ in diesem Jahr mit dem Robert Koch Award prämiert.

Weitere Informationen bei

Medizin \& Markt GmbH

Pia Nothing

nothing@medizin-und-markt.de
Falk Foundation e.V. In der neuen von der Falk Foundation herausgegebenen Patientenbroschüre «Darmkrebserkrankungen» werden wichtige Fragen zu Dickdarm- und Enddarmkrebs beantwortet. Die Broschüre «Darmkrebserkrankungen» kann kostenfrei unter dem Code S94 bei folgender Adresse bestellt werden:

Falk Foundation e.V.

Leinenweberstr. 5

79108 Freiburg

Fax +497611514 321

literaturservice@falkfoundation.de
Wyeth Pharma GmbH. Tygaci ${ }^{\circledR}$ (Tigecyclin) wirkt effektiv gegen die Erreger von komplizierten Infektionen des Abdomens sowie der Haut und des Weichgewebes bei hospitalisierten Patienten mit erheblichen Komorbiditäten. Dieses belegen Ergebnisse einer multizentrischen nichtinterventionellen Studie (Eckmann C. et al.: Posterpräsentation DIVI 2008, Hamburg).

Weitere Informationen be

Medizin \& Markt GmbH

Ingrid Wilke

Tel. +49 89 383930-36

wilke@ medizin-und-markt.de 